\title{
Local radiation for cutaneous T-cell lymphoma other than mycosis fungoides and Sézary syndrome
}

\author{
Naoto Shikama \\ Department of Radiation Oncology, Juntendo University Graduate School of Medicine, 2-1-1 Hongo, Bunkyo-ku, Tokyo 113-8421 Japan \\ Correspondence to: Naoto Shikama, MD, PhD. Department of Radiation Oncology, Juntendo University Graduate School of Medicine, 2-1-1 Hongo, \\ Bunkyo-ku, Tokyo 113-8421 Japan. Email: nshikama0525@gmail.com.
}

\begin{abstract}
Primary cutaneous lymphoma is the second most common type of extranodal lymphoma. The clinical behavior of this lymphoma differs from that of other extranodal lymphomas and thus requires a particular pretreatment evaluation and treatment strategy. Cutaneous T-cell lymphoma (CTCL) accounts for $80 \%$ of primary cutaneous lymphoma cases and includes several confirmed disease entities as well as provisional entities. Local radiation for CTCLs is applicable for both curative and palliative intents and is based on the involved-site radiotherapy (ISRT) concept. Primary cutaneous CD30-positive lymphoproliferative disorders include primary cutaneous anaplastic large-cell lymphoma (C-ALCL), lymphomatoid papulosis (LyP), and borderline lesions, all of which exhibit indolent behavior. One half of all LyP cases show spontaneous regression and do not require active treatment. Solitary or localized C-ALCL cases are treated with surgical excision or local radiation of 24-36 Gy. The most common relapse site after local treatment is other skin areas; however, skin relapse is not associated with worsened prognosis. Subcutaneous panniculitis-like T-cell lymphoma (SPTL) without hemophagocytic syndrome exhibits indolent behavior, and localized lesions can be successfully treated with local radiation of 40 Gy or more. Extranodal NK/T-cell lymphoma, nasal type has an aggressive clinical behavior even with intensive chemotherapy and a dose of 50 Gy or higher might be required for good tumor control. Palliative local radiation of $8 \mathrm{~Gy}$ in one or two fractions is effective in treating refractory or relapsed CTCLs. ISRT with curative intent should encompass the original suspicious lesions plus a 2-3 cm margin, while ISRT with palliative intent should encompass visible lesions plus a 1-2 cm margin. Appropriate electron beam energy and bolus thickness should be selected according to the skin surface dose and thickness of the patch and plaque lesions. All CTCL cases should be reviewed by a multidisciplinary team to ensure an appropriate treatment strategy.
\end{abstract}

Keywords: Cutaneous T-cell lymphoma (CTCL); CD30-positive lymphoproliferative disorders; subcutaneous panniculitis-like T-cell lymphoma (SPTL); extranodal NK/T-cell lymphoma; nasal type; local radiation

Submitted May 19, 2018. Accepted for publication Jun 28, 2018.

doi: 10.21037/cco.2018.07.01

View this article at: http://dx.doi.org/10.21037/cco.2018.07.01

\section{Introduction}

Primary cutaneous lymphoma is the second most common type of extranodal lymphoma, and is defined as a hematological neoplasm present in the skin with no evidence of extra-cutaneous disease at the time of diagnosis (1). It exhibits significantly different clinical behavior and prognosis from those of extranodal lymphomas with origins other than the skin, and each requires a particular treatment strategy. Primary cutaneous T-cell lymphoma (CTCL) occurs most frequently in patients in their 60 s to 80s (predominantly in males) and accounts for $71-87 \%$ of primary cutaneous lymphomas (2-4). The Surveillance, Epidemiology, and End Results (SEER) program deems that CTCL incidence is 10 per million persons, the proportion of which has been stable since 1998 (2). The World Health 
Organization (WHO) and the European Organization for Research and Treatment of Cancer (EORTC) reported new classification for primary cutaneous lymphomas in 2005, and the disease concepts of primary cutaneous lymphoma have been dramatically reorganized since then (1). CTCL cases primarily comprise those involving mycosis fungoides, Sézary syndrome, primary cutaneous CD30-positive lymphoproliferative disorders, subcutaneous panniculitislike T-cell lymphoma (SPTL), extranodal NK/T-cell lymphoma, nasal type (ENKTCL), adult T-cell lymphoma, and primary cutaneous peripheral T-cell lymphoma, unspecified (PTCL-NOS). The 2016 revision of the WHO classification of lymphoid neoplasms added two provisional disease entities of CTCLs (5). Over the past decade, the international consensus of staging classification, clinical endpoints and response criteria, and treatment guidelines for CTCLs have been reported, and basic systems to establish a standard of care for CTCLs have progressed (1,6-14). Local radiation for CTCLs has been applied with both curative and palliative intents in clinical practice (15). The aim of this article is to clarify the utility of local radiation for CTCLs other than mycosis fungoides and Sézary syndrome as part of a multidisciplinary treatment strategy.

\section{Pretreatment evaluation}

Biopsies of all types (punch, incisional, or excisional biopsy) of suspicious skin lesions aid in the final diagnosis, and all specimen slides should be reviewed by experienced hematopathologists or dermatopathologists (16). Rebiopsy should be considered if the histopathological specimen is insufficient to make a final diagnosis. Adequate immunohistochemistry panels for final diagnosis may include CD2, CD3, CD4, CD8, CD20, CD30, CD56, anaplastic lymphoma kinase, BCL6, Ki-67, and other typing panels (16). Clonal T-cell receptor gene rearrangement including alpha/beta $(\alpha / \beta)$ and gamma/delta $(\gamma / \delta)$ chains is essential for making a final diagnosis and deciding on a treatment strategy (1).

The International Society for Cutaneous Lymphomas (ISCL) and EORTC revised the TNMB staging system and classification for mycosis fungoides and Sézary syndrome in 2007 (17). They also proposed a separate TNM staging system for CTCLs other than mycosis fungoides and Sézary syndrome (12). All patients should undergo the appropriate staging procedures, which include obtaining a medical history, complete physical examination including peripheral lymph node regions, liver and spleen enlargement, complete skin examination by a dermatologist, complete blood count with differential white blood cell count, serum biochemistry including lactate dehydrogenase, and imaging evaluation $(9,11,16,17)$. Standardized photographs are recommended for documentation of the appearance of skin lesions at baseline and during disease progression (11). Contrast-enhanced computed tomography (CT) of the chest, abdomen, and pelvis is routinely undertaken for patients with T3-4 disease to assess nodal and visceral involvement, but has limited value for patients with T1-2 disease $(13,18)$. However, unlike mycosis fungoides, PTCL-NOS does not follow the typical progression process through localized patch and plaque disease via tumor-stage disease and finally to generalized and/or disseminated disease. Instead, PTCL-NOS is more likely to present as widespread plaque disease coexisting with tumor-stage disease from the onset (19). Integrated whole body fludeoxyglucose $\left({ }^{18} \mathrm{~F}\right)$ positron emission tomography and CT are recommended for patients with primary cutaneous anaplastic large-cell lymphoma (C-ALCL) and more aggressive CTCLs such as ENKTCL and PTCLNOS $(16,20)$. Bone marrow aspiration and biopsy have limited value for patients with C-ALCL, but may prove to be informative for patients with ENKTCL and PTCL-NOS. Examination of the ear, nose, and throat should be taken into consideration for patients with ENKTCL (16).

\section{Local radiation for primary cutaneous CD30- positive lymphoproliferative disorders}

Primary cutaneous CD30-positive lymphoproliferative disorders are the second most common type of CTCLs, and account for $14-30 \%$ of all CTCLs $(1,4)$. This particular type consists of C-ALCL, lymphomatoid papulosis (LyP), and borderline lesions $(10,21)$. These disease entities occur on a continuous disease spectrum ranging from benign $\mathrm{Ly} P$ to malignant C-ALCL, with substantial overlap between entities (21-24). Histological differentiation between C-ALCL and LyP is difficult, and clinicopathological correlation is critical to make an accurate diagnosis $(25,26)$. History and evidence of other CTCLs should be evaluated at the initial diagnosis $(25,27)$. C-ALCL is defined as a CTCL composed of anaplastic large cells with expression of CD30 in more than $75 \%$ of tumor cells (28). C-ALCL usually appears on the extremities, trunk, and head and neck, and affects middle-age and elderly patients (predominantly males) $(21,23,28-30)$. Eighty percent of patients with C-ALCL have solitary or localized lesions, and $20 \%$ have generalized skin lesions (31). Extra- 
cutaneous involvement occurs in approximately $10 \%$ of patients with C-ALCL, and tends to affect regional lymph nodes (21). Spontaneous regression ranging from 6 to $22 \%$ has been observed in patients with C-ALCL, and remaining lesions and regrowth lesions require active treatment $(26,32)$. For solitary and localized C-ALCL, waiting for spontaneous regression, surgical excision, or local radiation represent first-line treatment options, while postoperative radiation may be considered for those with insufficient surgical margins $(24,27,32)$. Although C-ALCL exhibits indolent behavior, localized involvement of the extremities with multiple lesions is known as "extensive limb disease" and is associated with poor outcomes (32). Patients with multiple or disseminated disease are treated with low-dose methotrexate, but intensive multi-agent chemotherapy is not usually recommended (28).

LyP usually occurs in middle-age patients but can also occur occasionally in elderly patients and children, on the trunk and extremities (21). Eighty percent of $\mathrm{LyP}$ cases show generalized skin lesions, with the remaining $20 \%$ showing localized skin lesions at presentation $(24,33)$. LyP has a histological appearance of a malignant disease, but exhibits clinically benign behavior (21). LyP is histologically heterogeneous with several histologic subtypes (types A to $\mathrm{D}$, among others) $(1,32,33)$. One half of LyP cases show spontaneous regression within weeks to months; these cases do not require active treatment $(2,21,27,32-34)$. Ten to $61 \%$ of patients with LyP develop or have coexisting hematologic malignancies including systemic anaplastic large cell lymphoma, mycosis fungoides, or Hodgkin's lymphoma $(26,27,34)$. Furthermore, $10 \%$ of patients with $\mathrm{LyP}$ have an increased risk of non-hematologic malignancies (35). Topical therapies and phototherapy provide early clinical response, but do not prevent the development of new lesions on the skin outside of the treated area $(32,34)$. No treatment has been developed that effectively influences the natural course of $\mathrm{LyP}$, and the short-term benefits of active treatment should be balanced carefully with potential longterm adverse effects (1). Localized LyP could be treated with close observation, topical steroids, and phototherapy as first-line treatment $(16,24)$. Close observation without radiation is an important strategy to prevent the development of secondary malignancies in patients with LyP (32). Phototherapy, surgical excision, or local radiation may be recommended as alternatives to observation for $\mathrm{LyP}$ lesions larger than $2 \mathrm{~cm}$ in diameter which persist for months without spontaneous regression (10,24). For generalized LyP lesions, close observation, low-dose methotrexate
(5-20 mg per week), phototherapy, systemic retinoids, topical steroids, or topical mechlorethamine have been reported $(16,32,33)$.

In 2011, the EORTC, ISCL, and United States Cutaneous Lymphoma Consortium (USCLC) reported an optimal radiation dose of 30-46 Gy for C-ALCL (10). However, one study of a Dutch registry of primary cutaneous lymphomas and one from the MD Anderson Cancer Center recently reported that there was no definite dose-response relationship for C-ALCL $(30,31)$. The former recommended 20 Gy in 8 fractions as the optimal regimen, while the latter recommended conventional fractionated radiation of $30 \mathrm{~Gy}$ or less. The International Lymphoma Radiation Oncology Group (ILROG) analyzed 56 patients recruited from the 8 collaborating institutions and reported a recommended dose of 30 Gy for good local control (29). The National Comprehensive Cancer Network (NCCN) Clinical Practice Guideline in Oncology (version 3. 2018) recommended a total dose of 24-36 Gy for C-ALCL (16). On the other hand, small case-series studies have applied fractionated radiotherapy of 30-45 Gy in 2-2.5 Gy fractions or a single fraction of 7.5-8 Gy for LyP $(22,36)$.

In most patients with localized C-ALCL, surgical resection or local radiation results in a complete response and excellent tumor control (23,25,27-29). Smith and colleagues reported that only $70 \%$ of patients achieved a complete response within the first 3 months after local radiation, but that after long-term follow-up most patients achieved excellent tumor control (30). Long-term careful observation after local radiation might be required to avoid over-treatment. Relapse rates after initial local treatment range from $30 \%$ to $64 \%$, with a median disease-free survival time of only 16 months $(23,25-27,32,37)$. The most common relapse site after local treatment is other skin areas (the same anatomic area as the initial lesion), while the next most common relapse site is the regional lymph node area $(1,23,27,30,32,37)$. Cutaneous relapse after local treatment is not associated with worsened prognosis. Ten-year diseasespecific survival rates of patients with localized C-ALCL range from $90-98 \%$, with a 5 -year disease-specific survival rate of approximately $50 \%$ among patients with generalized lesions $(1,26,28,29,34)$.

In a recent multicenter retrospective analysis of 252 patients with LyP, complete response to first-line treatment including topical steroids, low-dose methotrexate, and phototherapy was achieved in only $48 \%$ of patients (33). Of those who achieved a complete response, $78 \%$ developed cutaneous relapse with a median disease-free survival of 
only 11 months (33). Type A lesions and use of first-line treatments other than phototherapy were associated with early cutaneous relapse. In spite of high cutaneous relapse rates after first-line treatment, $\mathrm{LyP}$ patients have excellent prognoses, with 5 -year overall survival rates of $98-100 \%$ $(1,32,34)$. Only $4 \%$ of patients with LyP developed systemic disease, and only $2 \%$ reportedly died of systemic disease within the median follow-up period of 6 years $(1,27)$. Regular, long-term follow-up is important to monitor development of second lymphoid and non-lymphoid malignancies (16). Patients with borderline lesions tend to develop C-ALCL more readily than patients with LyP, but the treatment outcome is favorable (21).

\section{Local radiation for SPTL}

SPTL is an extremely rare disease, accounting for approximately $2 \%$ of CTCL cases in Japan (4). According to the SEER program, SPTL affects middle-aged (median age, 47 years) individuals, predominantly female (67\%) (38). Previously, confusion surrounded the disease entity of SPTL until the new WHO-EORTC classification was reported in 2005. Currently, the term "SPTL" is only used for patients with $\alpha / \beta$ T-cell phenotype $(1,39,40)$. CTCLs with $\gamma / \delta$ T-cell phenotype should be excluded from the current definition. SPTL with $\alpha / \beta$ T-cell phenotype generally presents as a CD4-, CD8+, CD56-, and $\beta \mathrm{F} 1+$ phenotype, and is uncommonly affiliated with hemophagocytic syndrome $(17-32 \%)(40,41)$. Patients show nodular skin lesions and deeply seated plaques, and half of them complain of fever, chills, night sweats, fatigue, and anorexia $(24,39,40)$. Most patients have generalized skin lesions in the extremities and trunk, and approximately $20 \%$ of patients have solitary or localized skin lesions. SPTL without hemophagocytic syndrome exhibits indolent behavior and excellent prognosis relative to that of SPTL with hemophagocytic syndrome (5-year overall survival: $91 \%$ vs. $46 \%$, respectively) $(9,40)$. Hemophagocytic syndrome is often associated with aggressive clinical behavior, and patients should be treated with intensive multi-agent chemotherapy $(1,9,13,40,41)$. Other unfavorable prognostic factors for overall survival of patients with SPTL include old age ( $>65$ years) and disseminated disease (38). Solitary or localized lesions of SPTL without hemophagocytic syndrome may be treated with local radiation alone $(9,39,40,42)$. CTCLs with $\gamma / \delta$ T-cell phenotype present as a CD4-, CD8-, CD56+/-, $\beta \mathrm{F} 1+$ phenotype, and are often affiliated with hemophagocytic syndrome and aggressive behavior $(1,39,40)$. Most patients with $\gamma / \delta$ T-cell phenotype have generalized lesions in the extremities and trunk, with approximately $30 \%$ of them displaying general symptoms and/or hepatosplenomegaly (40). Patients with $\gamma / \delta$ T-cell phenotype are treated with intensive multi-agent chemotherapy, but the clinical response to chemotherapy has been disappointing (41). Systemic relapse has been observed in the lungs, liver, kidneys, and central nervous system, and high-dose chemotherapy and stem cell transplantation have been investigated (41).

Although there is little information on the optimal radiation dose for SPTL, the European Society for Medical Oncology (ESMO) and ILROG recommend high-dose radiation of $40 \mathrm{~Gy}$ or more $(8,42)$. In one study, after initial treatment, half of those with $\alpha / \beta$ T-cell phenotype developed new skin lesions outside the irradiated areas, but none developed extra-cutaneous involvement (40). Kong and colleagues reported that approximately half of their patients showed complete or partial responses after multiagent chemotherapy (39). The 5 -year overall survival rate of patients with current SPTL might be more than $80 \%$, while that of patients with $\gamma / \delta$ T-cell phenotype is only $11 \%$ $(1,9,38-40)$.

\section{Local radiation for extranodal NK/T-cell Iymphoma, nasal type (ENKTCL)}

ENKTCL is nearly always Epstein-Barr virus-related lymphoma, and is relatively common in Asia, Central America, and South America $(1,21,42)$. A nationwide survey by the Japanese Skin Cancer Society Lymphoma Study Group found that among 1,733 patients with primary cutaneous lymphomas, the incidence of ENKTCL was $2.3 \%$ (4), which was higher than that in Western countries $(<1 \%)$, but lower than that in Korea (20\%). Multiple subcutaneous nodules with or without necrosis are found in the extremities, head and neck, and trunk $(21,43)$. ENKTCL shows CD56 positivity, and exhibits aggressive clinical behavior and poor survival rates $(9,21,43)$. Although intensive multi-agent chemotherapy could be applied, resistance to anthracycline-containing chemotherapy is common (43). Patients with advanced disease could be treated with platinum-based chemotherapy with or without local radiation, but thus far, treatment outcomes have been disappointing $(1,13)$.

High-dose radiation to the primary site might be required for good tumor control, but the role of local radiation has not been clarified for patients with generalized 
ENKTCL lesions. The NCCN Clinical Practice Guideline recommends a radiation dose of 45-50.4 Gy when combination therapy is applied, and 50 Gy or higher with radiotherapy alone (16). ILROG and ESMO recommended a dose of 50 Gy to the initial lesion followed by boost irradiation of 5-10 Gy to residual lesions $(8,42)$. Median survival time of patients with cutaneous localized lesions is 27 months, and that of patients with extra-cutaneous disseminated lesions ranges from 5 to 10 months $(1,43)$.

\section{Local radiation for primary cutaneous peripheral T-cell lymphoma, unspecified (PTCL-NOS)}

PTCL-NOS is a heterogeneous group that includes CTCL types that do not fit into any of the better defined subtypes of the WHO-EORTC classification; it also includes several ill-defined provisional subtypes $(1,21)$. A careful clinical history and accurate clinical examination are required to rule out transformed mycosis fungoides and other CTCLs (28). PTCL-NOS accounts for $6.7 \%$ of CTCLs in Japan (4). Each provisional subtype accounts for less than $1 \%$ of CTCLs, and their clinical behaviors have not been clarified. PTCL-NOS generally presents with an aggressive clinical behavior and poor survival rate, and commonly occurs in adults $(1,9)$. Primary cutaneous aggressive epidermotropic CD8+ T-cell lymphoma and cutaneous $\gamma / \delta$ T-cell lymphoma present with progressive behavior, and even patients with solitary or localized lesions rapidly develop widespread disease $(1,44)$. Primary cutaneous aggressive epidermotropic CD8+ T-cell lymphoma has localized or disseminated lesions with ulceration, hemorrhage, and necrosis, and tends to disseminate to other visceral organs including the lungs, testes, oral cavity, and central nervous system, but generally spares the lymph nodes (19). CD8 positivity itself is not associated with a poor prognosis, and among CD8-positive patients with mycosis fungoides variant, C-ALCL and other CTCLs exhibit indolent behavior (45). Discerning between primary cutaneous aggressive epidermotropic CD8+ T-cell lymphoma and other CD8-positive CTCLs is critical in order to avoid overly aggressive treatment. Furthermore, the 2016 revision of the WHO classification of lymphoid neoplasms proposed "Primary cutaneous acral CD8+ T-cell lymphoma" as a provisional entity; this lymphoma exhibits indolent clinical behavior (5).

Primary cutaneous $\gamma / \delta$ T-cell lymphoma presents as generalized skin lesions on the extremities $(1,21)$. Intensive systemic chemotherapy can be applied, but the clinical response has been disappointing $(44,46)$. Allogeneic stem cell transplantation can be considered for some cases, but little information is available on anticipated outcomes (42).

Half of the patients with primary cutaneous CD4+ small/ medium-sized pleomorphic T-cell lymphoma have solitary or localized skin lesions, and others have multifocal lesions $(44,47)$. Those with a solitary lesion and low proliferative index (Ki-67 <10\%) can expect excellent outcomes after local radiation or surgical excision $(42,44,47)$. For patients with disseminated disease, intermediate levels of aggression are observed, and relapse and progression to the lymph nodes and visceral organs can develop after multi-agent chemotherapy (21). Primary cutaneous CD4+ small/ medium-sized pleomorphic T-cell lymphoma with a high proliferative index (Ki-67 >20\%) and/or bulky tumors exhibit aggressive clinical behavior (47). Differential diagnosis between the two types of primary cutaneous CD4+ small/medium-sized pleomorphic T-cell lymphomas is critical for planning an appropriate treatment strategy.

ILROG reported that a clinical response for primary cutaneous $\gamma / \delta$ T-cell lymphoma after conventional fractionated local radiation of 24-30 Gy may be possible, but that it tends to relapse at the same site $(8,46)$. Pedretti and colleagues treated 10 patients with primary cutaneous $\gamma / \delta$ T-cell lymphoma using 30-40 Gy (median, 36 Gy), and found that 3 patients developed local relapses at the same site (46). The NCCN Clinical Practice Guideline provides general radiation dose guidance for peripheral T-cell lymphoma arising from any site, and recommends a consolidation dose for complete response after chemotherapy of 30-36 Gy, a complementary dose for partial response of 40-50 Gy, and a dose of 40-55 Gy for primary treatment of non-candidates for chemotherapy or as salvage treatment for refractory disease (16).

Median survival times of patients with primary cutaneous aggressive epidermotropic CD8+ T-cell lymphoma and cutaneous $\gamma / \delta$ T-cell lymphoma are 32 months and 15 months, respectively $(1,21)$. Patients with primary cutaneous CD4+ small/medium-sized pleomorphic T-cell lymphoma have a relatively favorable prognosis, with 5-year overall survival rates of $75 \%$ (1). Among patients with small/ medium-sized pleomorphic T-cell lymphoma, those with a CD4-positive phenotype are likely to have a good prognosis relative to those with a CD4-negative phenotype (44).

\section{Palliative local radiation for refractory or relapsed CTCL}

Short-course local radiation is an effective palliative therapy 


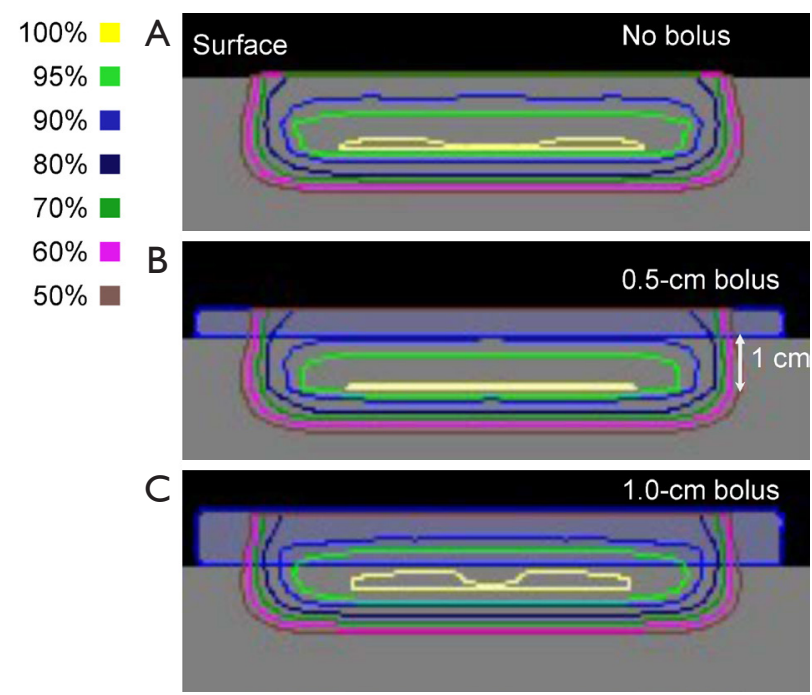

Figure 1 Dose distribution using $6 \mathrm{MeV}$ electron beams with/ without bolus on smooth surface site of rectangular phantom. (A) Surface dose is approximately $80 \%$ (without bolus); (B) Bolus of $0.5-\mathrm{cm}$ thickness improves the surface dose to approximately $90 \%$; (C) Bolus of 1-cm thickness improves the surface dose to approximately $100 \%$.

for refractory or relapsed CTCLs $(22,48-50)$. Although the clinical response after 4 Gy in 2 fractions is only $30 \%$ and remission time is very short for symptomatic CTCLs, symptom relief after 8 Gy in 1 or 2 fractions is favorable, with a complete response rate over $90 \%$ and a mean remission time for relapse of approximately 9 months $(8,48,49,51)$. Bulky tumors may require a higher dose (8). For primary cutaneous CD30-positive lymphoproliferative disorders, a single fraction of 7.5-8 Gy results in a $100 \%$ complete response rate and long remission (22). Thomas and colleagues analyzed 58 patients with CTCLs (mycosis fungoides in 47 patients; LyP in 4; cutaneous $\gamma / \delta$ T-cell lymphoma in 3; Sézary syndrome in 3) who were treated by a single fraction of palliative radiation, and reported a complete response rate of $94 \%$ after a single fraction of $7-$ 8 Gy (49). However, the clinical response of CD4-negative lesions after local radiation was disappointing (40-50\%) when compared to that of CD4-positive lesions (49). Small case-series studies found complete response rates ranging from $50 \%$ to $100 \%$ after $6-8$ Gy in 2-4 Gy fractions $(52,53)$. Initial radiation doses of 8 to 12 Gy will allow reirradiation, although a smaller fraction size of 3-5 Gy might be preferred for re-irradiation (8). Fractionated radiation should be considered in cases involving a large radiation field $(8,34)$. The appropriate palliative radiation dose for ENKTCL has not been clarified, but high-dose radiation might be required to obtain good symptom relief and tumor control.

\section{Technical aspects of local radiation}

Local radiation for primary cutaneous lymphoma is based on the ISRT concept, which minimizes the prophylactic irradiated area without compromising treatment outcome (16). It spares adjacent uninvolved organs such as lungs, bone, and muscle. Clinical target volume (CTV) for patients with patch and/or plaque disease should encompass the epidermis and dermis, and that for patients with tumor lesions should encompass the tumor extension $(34,54)$. The lateral field margin of definitive local radiation can be limited to $2-3 \mathrm{~cm}$ beyond the pretreatment defined lesion and suspicious area $(8,34,55)$. For palliative radiotherapy, CTV will encompass the visible lesion with a margin of 1$2 \mathrm{~cm}$ of surrounding healthy-looking skin $(8,48)$. Appropriate electron beam energy should be selected for the skin surface dose and thickness of patch and plaque lesions, and photon beams could be considered for tumor lesions. A daily bolus of $0.5-1 \mathrm{~cm}$ thickness is used to prescribe to the $80-90 \%$ isodose line to cover each lesion (55) (Figure 1).

A single radiation field of electron beam is desirable from the viewpoint of homogeneous dose distribution, but the multiple-field technique is required at complex anatomical sites such as the face, axilla, and breast, and at convex surface sites such as the scalp and extremities. If the multiple-field technique using electron beams is applied, then the junction of abutting fields should be shifted frequently during the course of treatment to avoid over-dose and under-dose in these areas (Figure 2A). A novel radiotherapy technique called helical intensitymodulated radiation therapy (helical IMRT), using 6 MV photons and binary collimators, might be useful for obtaining homogeneous dose distribution at complex anatomical sites and convex surface sites (Figure 2B). The advantages of helical IMRT are (I) homogeneous dose distribution to the skin and subcutaneous area, (II) irregular-shaped radiation field to avoid risk organs, and (III) sparing of deep structures such as bone marrow and visceral organs. Meanwhile, disadvantages include the (I) complex technique and (II) inconvenience of plan modification upon tumor shrinkage. Another radiation technique using photon beams with rice packing for tissue compensation could be applicable for highly irregular surface sites such as the 

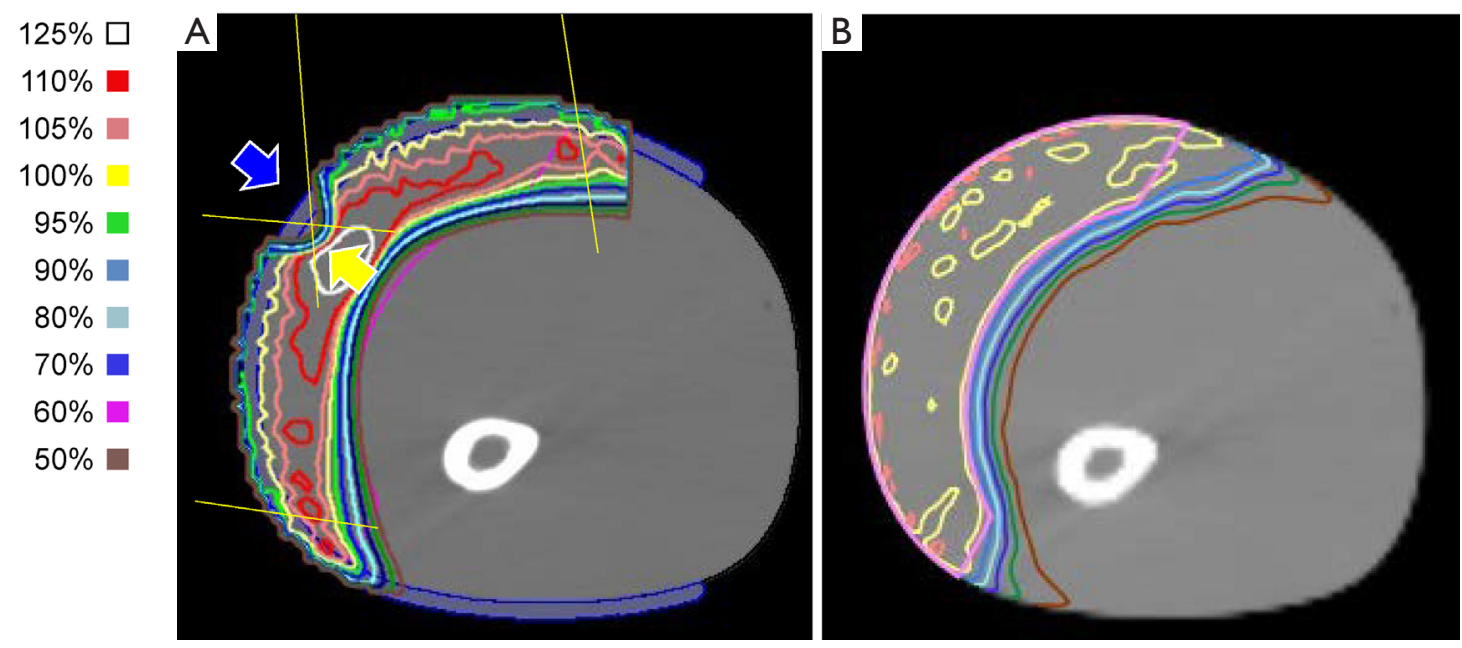

Figure 2 Dose distribution on convex surface of lower limb phantom by the multiple-field technique of electron beams and helical intensitymodulated radiation therapy (helical IMRT). (A) Multiple-field technique using $9 \mathrm{MeV}$ electron beams creates low-dose and over-dose areas in the junction of abutting fields. Blue arrow: low-dose area on the skin surface. Yellow arrow: high-dose area in the subcutaneous area. (B) Helical IMRT using $6 \mathrm{MV}$ photons and bolus is able to deliver homogeneous dose distribution over a large convex surface area. High-dose areas of more than $110 \%$ are not shown.

entire foot (including digits) (56). The advantages of the rice packing technique include the (I) simple work process and (II) homogeneous dose distribution regardless of tumor shrinkage. Disadvantages include the (I) high radiation dose to deep structures, (II) difficulty of creating an irregular-shaped radiation field to avoid irradiation of risk organs, and (III) applicational difficulty for head and neck irradiation. A small case-series study reported the utility of a brachytherapy technique for CTCL lesions arising from convex surfaces such as the hands or feet (50).

\section{Conclusions}

All patient cases should be reviewed by a multidisciplinary team to ensure the selection of an appropriate treatment strategy based on histological subtype, staging evaluation, clinical features, and patient condition. Further prospective and retrospective studies and a large-scale registry database are required to establish the standard of care for CTCLs.

\section{Acknowledgements}

I would like to thank Mr. K. Usui for his technical support. Funding: This work was supported by a Grant-in-Aid for Cancer Research from the Ministry of Health, Labour and Welfare of Japan (29-A-3).

\section{Footnote}

Conflicts of Interest: The author has no conflicts of interest to declare.

\section{References}

1. Willemze R, Jaffe ES, Burg G, et al. WHO-EORTC classification for cutaneous lymphomas. Blood 2005;105:3768-85.

2. Korgavkar K, Xiong M, Weinstock MA. Compact fluorescent lamps and risk of skin cancer. J Cutan Med Surg 2013;17:308-12.

3. Korgavkar K, Weinstock MA. Changing incidence trends of cutaneous B-cell lymphoma. J Invest Dermatol 2014;134:840-2.

4. Hamada T, Iwatsuki K. Cutaneous lymphoma in Japan: a nationwide study of 1733 patients. J Dermatol 2014;41:3-10.

5. Swerdlow SH, Campo E, Pileri SA, et al. The 2016 revision of the World Health Organization classification of lymphoid neoplasms. Blood 2016;127:2375-90.

6. Trautinger F, Knobler R, Willemze R, et al. EORTC consensus recommendations for the treatment of mycosis fungoides/Sezary syndrome. Eur J Cancer 2006;42:1014-30.

7. Trautinger F, Eder J, Assaf C, et al. European Organisation for Research and Treatment of Cancer consensus recommendations for the treatment of mycosis 
fungoides/Sezary syndrome - Update 2017. Eur J Cancer 2017;77:57-74.

8. Specht L, Dabaja B, Illidge T, et al. Modern radiation therapy for primary cutaneous lymphomas: field and dose guidelines from the International Lymphoma Radiation Oncology Group. Int J Radiat Oncol Biol Phys 2015;92:32-9.

9. Willemze R, Dreyling M, Group EGW. Primary cutaneous lymphomas: ESMO Clinical Practice Guidelines for diagnosis, treatment and follow-up. Ann Oncol 2010;21 Suppl 5:v177-80.

10. Kempf W, Pfaltz K, Vermeer MH, et al. EORTC, ISCL, and USCLC consensus recommendations for the treatment of primary cutaneous CD30-positive lymphoproliferative disorders: lymphomatoid papulosis and primary cutaneous anaplastic large-cell lymphoma. Blood 2011;118:4024-35.

11. Olsen EA, Whittaker S, Kim YH, et al. Clinical end points and response criteria in mycosis fungoides and Sezary syndrome: a consensus statement of the International Society for Cutaneous Lymphomas, the United States Cutaneous Lymphoma Consortium, and the Cutaneous Lymphoma Task Force of the European Organisation for Research and Treatment of Cancer. J Clin Oncol 2011;29:2598-607.

12. Kim YH, Willemze R, Pimpinelli N, et al. TNM classification system for primary cutaneous lymphomas other than mycosis fungoides and Sezary syndrome: a proposal of the International Society for Cutaneous Lymphomas (ISCL) and the Cutaneous Lymphoma Task Force of the European Organization of Research and Treatment of Cancer (EORTC). Blood 2007;110:479-84.

13. Whittaker SJ, Marsden JR, Spittle M, et al. Joint British Association of Dermatologists and U.K. Cutaneous Lymphoma Group guidelines for the management of primary cutaneous T-cell lymphomas. Br J Dermatol 2003;149:1095-107.

14. Scarisbrick JJ, Prince HM, Vermeer MH, et al. Cutaneous Lymphoma International Consortium Study of Outcome in Advanced Stages of Mycosis Fungoides and Sezary Syndrome: Effect of Specific Prognostic Markers on Survival and Development of a Prognostic Model. J Clin Oncol 2015;33:3766-73.

15. Shikama N, Miyazawa K. Cutaneous lymphoma. Radiation Therapy for Extranodal Lymphomas. Tokyo: Springer; 2017.

16. T-Cell Lymphomas. Version 3.2018 NCCN Clinical Practice Guidelines in Oncology. 2018.
17. Olsen E, Vonderheid E, Pimpinelli N, et al. Revisions to the staging and classification of mycosis fungoides and Sezary syndrome: a proposal of the International Society for Cutaneous Lymphomas (ISCL) and the cutaneous lymphoma task force of the European Organization of Research and Treatment of Cancer (EORTC). Blood 2007;110:1713-22.

18. Hwang ST, Janik JE, Jaffe ES, et al. Mycosis fungoides and Sezary syndrome. Lancet 2008;371:945-57.

19. Gormley RH, Hess SD, Anand D, et al. Primary cutaneous aggressive epidermotropic CD8+ T-cell lymphoma. J Am Acad Dermatol 2010;62:300-7.

20. Lee WJ, Lee YJ, Won CH, et al. The applicability and prognostic value of the TNM classification system for primary cutaneous lymphomas other than mycosis fungoides and Sezary syndrome in primary cutaneous NK/T-cell lymphoma patients. J Am Acad Dermatol 2015;73:172-4 e1.

21. Paulli M, Berti E. Cutaneous T-cell lymphomas (including rare subtypes). Current concepts. II. Haematologica 2004;89:1372-88.

22. Gentile MS, Martinez-Escala ME, Thomas TO, et al. Single-Fraction Radiotherapy for CD30(+) Lymphoproliferative Disorders. Biomed Res Int 2015;2015:629587.

23. Beljaards RC, Kaudewitz P, Berti E, et al. Primary cutaneous CD30-positive large cell lymphoma: definition of a new type of cutaneous lymphoma with a favorable prognosis. A European Multicenter Study of 47 patients. Cancer 1993;71:2097-104.

24. Siegel RS, Pandolfino T, Guitart J, et al. Primary cutaneous T-cell lymphoma: review and current concepts. J Clin Oncol 2000;18:2908-25.

25. Yu JB, McNiff JM, Lund MW, et al. Treatment of primary cutaneous CD30+ anaplastic large-cell lymphoma with radiation therapy. Int J Radiat Oncol Biol Phys 2008;70:1542-5.

26. Liu HL, Hoppe RT, Kohler S, et al. CD30+ cutaneous lymphoproliferative disorders: the Stanford experience in lymphomatoid papulosis and primary cutaneous anaplastic large cell lymphoma. J Am Acad Dermatol 2003;49:1049-58.

27. Bekkenk MW, Geelen FA, van Voorst Vader PC, et al. Primary and secondary cutaneous CD30(+) lymphoproliferative disorders: a report from the Dutch Cutaneous Lymphoma Group on the long-term followup data of 219 patients and guidelines for diagnosis and treatment. Blood 2000;95:3653-61. 
28. Quintanilla-Martinez L, Jansen PM, Kinney MC, et al. Non-mycosis fungoides cutaneous T-cell lymphomas: report of the 2011 Society for Hematopathology/European Association for Haematopathology workshop. Am J Clin Pathol 2013;139:491-514.

29. Million L, Yi EJ, Wu F, et al. Radiation Therapy for Primary Cutaneous Anaplastic Large Cell Lymphoma: An International Lymphoma Radiation Oncology Group Multi-institutional Experience. Int J Radiat Oncol Biol Phys 2016;95:1454-9.

30. Smith GL, Duvic M, Yehia ZA, et al. Effectiveness of lowdose radiation for primary cutaneous anaplastic large cell lymphoma. Adv Radiat Oncol 2017;2:363-9.

31. Melchers RC, Willemze R, Daniels LA, et al. Recommendations for the Optimal Radiation Dose in Patients With Primary Cutaneous Anaplastic Large Cell Lymphoma: A Report of the Dutch Cutaneous Lymphoma Group. Int J Radiat Oncol Biol Phys 2017;99:1279-85.

32. Wieser I, Tetzlaff MT, Torres Cabala CA, et al. Primary cutaneous CD30(+) lymphoproliferative disorders. J Dtsch Dermatol Ges 2016;14:767-82.

33. Fernandez-de-Misa R, Hernandez-Machin B, Servitje $\mathrm{O}$, et al. First-line treatment in lymphomatoid papulosis: a retrospective multicentre study. Clin Exp Dermatol 2018;43:137-43.

34. Smith BD, Wilson LD. Cutaneous lymphomas. Semin Radiat Oncol 2007;17:158-68.

35. Wang HH, Myers T, Lach LJ, et al. Increased risk of lymphoid and nonlymphoid malignancies in patients with lymphomatoid papulosis. Cancer 1999;86:1240-5.

36. Kaufmann TP, Coleman M, Nisce LZ. Ki-1 skin lymphoproliferative disorders: management with radiation therapy. Cancer Invest 1997;15:91-7.

37. Huang BS, Chen WY, Wang CW, et al. Relapse Pattern and Treatment Outcome of Curative Radiotherapy for Primary Cutaneous CD30+ Anaplastic Large-cell Lymphoma: A Retrospective Cohort Study. Acta Derm Venereol 2016;96:394-5.

38. Bhatt VR, Giri S, Verma V, et al. Survival of Subcutaneous Panniculitis-Like T-Cell Lymphoma and Peripheral T-Cell Lymphoma Not Otherwise Specified: A PropensityMatched Analysis of the Surveillance, Epidemiology, and End Results Database. Clin Lymphoma Myeloma Leuk 2016;16:373-8.

39. Kong YY, Dai B, Kong JC, et al. Subcutaneous panniculitis-like T-cell lymphoma: a clinicopathologic, immunophenotypic, and molecular study of 22 Asian cases according to WHO-EORTC classification. Am J Surg
Pathol 2008;32:1495-502.

40. Willemze R, Jansen PM, Cerroni L, et al. Subcutaneous panniculitis-like T-cell lymphoma: definition, classification, and prognostic factors: an EORTC Cutaneous Lymphoma Group Study of 83 cases. Blood 2008;111:838-45.

41. Go RS, Wester SM. Immunophenotypic and molecular features, clinical outcomes, treatments, and prognostic factors associated with subcutaneous panniculitis-like T-cell lymphoma: a systematic analysis of 156 patients reported in the literature. Cancer 2004;101:1404-13.

42. Willemze R, Hodak E, Zinzani PL, et al. Primary cutaneous lymphomas: ESMO Clinical Practice Guidelines for diagnosis, treatment and follow-up. Ann Oncol 2013;24 Suppl 6:vi149-54.

43. Chang SE, Huh J, Choi JH, et al. Clinicopathological features of CD56+ nasal-type T/natural killer cell lymphomas with lobular panniculitis. Br J Dermatol 2000;142:924-30.

44. Bekkenk MW, Vermeer MH, Jansen PM, et al. Peripheral T-cell lymphomas unspecified presenting in the skin: analysis of prognostic factors in a group of 82 patients. Blood 2003;102:2213-9.

45. Dummer R, Kamarashev J, Kempf W, et al. Junctional CD8+ cutaneous lymphomas with nonaggressive clinical behavior: a CD8+ variant of mycosis fungoides? Arch Dermatol 2002;138:199-203.

46. Pedretti S, Urpis M, Leali C, et al. Primary cutaneous non-Hodgkin lymphoma: results of a retrospective analysis in the light of the recent ILROG guidelines. Tumori 2018;104:394-400.

47. Garcia-Herrera A, Colomo L, Camos M, et al. Primary cutaneous small/medium CD4+ T-cell lymphomas: a heterogeneous group of tumors with different clinicopathologic features and outcome. J Clin Oncol 2008;26:3364-71.

48. Neelis KJ, Schimmel EC, Vermeer MH, et al. Lowdose palliative radiotherapy for cutaneous B- and T-cell lymphomas. Int J Radiat Oncol Biol Phys 2009;74:154-8.

49. Thomas TO, Agrawal P, Guitart J, et al. Outcome of patients treated with a single-fraction dose of palliative radiation for cutaneous T-cell lymphoma. Int J Radiat Oncol Biol Phys 2013;85:747-53.

50. Goddard AL, Vleugels RA, LeBoeuf NR, et al. Palliative Therapy for Recalcitrant Cutaneous T-Cell Lymphoma of the Hands and Feet With Low-Dose, High Dose-Rate Brachytherapy. JAMA Dermatol 2015;151:1354-7.

51. Kazmierska J. Clinical results of the total skin electron irradiation of the mycosis fungoides in adults. 
Conventional fractionation and low dose schemes. Rep Pract Oncol Radiother 2013;19:99-103.

52. Sina B, Burnett JW. Lymphomatoid papulosis. Case reports and literature review. Arch Dermatol 1983;119:189-97.

53. Scarisbrick JJ, Evans AV, Woolford AJ, et al. Regional lymphomatoid papulosis: a report of four cases. Br J Dermatol 1999;141:1125-8.

54. Jones GW, Kacinski BM, Wilson LD, et al. Total skin electron radiation in the management of mycosis fungoides: Consensus of the European Organization for Research and Treatment of Cancer (EORTC) Cutaneous
Lymphoma Project Group. J Am Acad Dermatol 2002;47:364-70.

55. Mazzeo E, Rubino L, Buglione M, et al. The current management of mycosis fungoides and Sezary syndrome and the role of radiotherapy: Principles and indications. Rep Pract Oncol Radiother 2013;19:77-91.

56. Majithia L, Rong Y, Siddiqui F, et al. Treating cutaneous T-cell lymphoma with highly irregular surfaces with photon irradiation using rice as tissue compensator. Front Oncol 2015;5:49.

Cite this article as: Shikama N. Local radiation for cutaneous T-cell lymphoma other than mycosis fungoides and Sézary syndrome. Chin Clin Oncol 2019;8(1):8. doi: 10.21037/ cсo.2018.07.01 